\title{
PAXgene Blood Tissue System
}

National Cancer Institute

\section{Source}

National Cancer Institute. PAXgene Blood Tissue System. NCI Thesaurus. Code C126392.

A system of proprietary reagents and supplies utilizing formalin-free solutions for the simultaneous preservation of histomorphology and biomolecules and the purification of RNA, DNA, miRNA, proteins, and phosphoproteins from the same sample. 